Conclusion: Hsa_circ_0012732, hsa_circ_0008961, hsa_circ_0405239 and hsa_circ_0068784 may be related to the pathogenesis of AS. Among them, hsa_circ_0012732 may be involved in AS inflammation and has the potential to participate in the judgment of disease activity.

REFERENCES:

[1] LS, K., et al., The biogenesis, biology and characterization of circular RNAs. 2019. 20(11): p. 675-691.

[2] J, W., et al., Non-coding RNAs in Rheumatoid Arthritis: From Bench to Bedside. 2019. 10: p. 3129

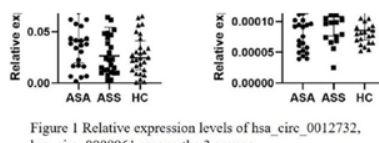

Figure 1 Relative expression levels of hsa_circ_0012732.
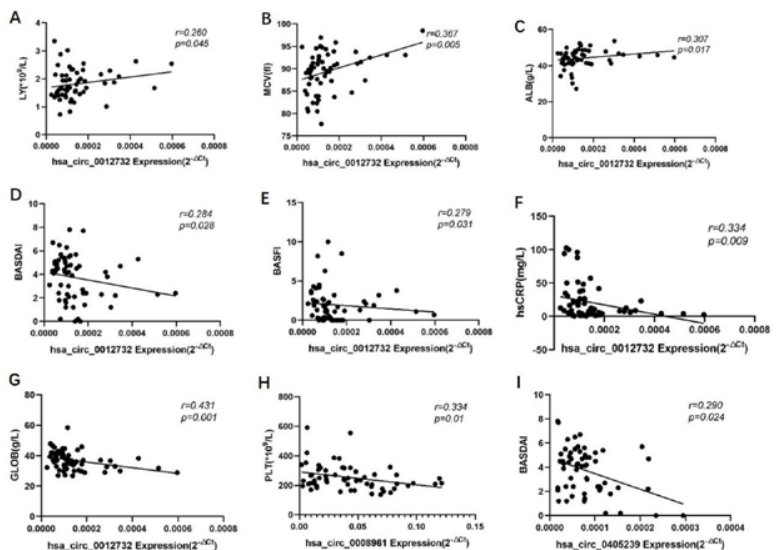

${ }^{3}{ }_{15}^{10}$.

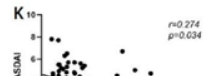

Disclosure of Interests: Yi-Ping Tang Grant/research support from: Science and Technology Project of Nanchong City (18SXHZ0522)., Yu-Feng Qing: None declared, Zeng-Rong Dong: None declared, Fei Dai: None declared, Jianxiong Zheng: None declared, Yi Jiang: None declared, Yi-Xi He: None declared, Quan-Bo Zhang Grant/research support from: National Natural Science Foundation of China(General Program) (81974250); Science and Technology Plan Project of Sichuan Province (2018JY0257)

DOI: 10.1136/annrheumdis-2021-eular.3412

\section{AB0076 \\ THE ROLE OF MACROPHAGE MIGRATION INHIBITORY FACTOR IN ACTIVITY AND PROGRESSION OF ANKYLOSING SPONDYLITIS}

Y. Gazar ${ }^{1}$, M. Hanafy ${ }^{1}$, M. Elrefi ${ }^{2} .{ }^{1}$ Faculty of Medicine, AL-Azhar university, Rheumatology and Rehabilitation, cairo, Egypt; ${ }^{2}$ Military Hospitals, Rheumatology, cairo, Egypt

Background: Ankylosing Spondylitis (AS) is a chronic inflammatory form of arthritis that predominantly affects the axial joints and has a global prevalence of $1 \%$. Disease progression is marked by osteoproliferation and bone fusion in the axial joints, causing considerable loss of mobility. Migration Inhibitory Factor (MIF) is a potent pro-inflammatory cytokine implicated in several diseases. Elevated serum MIF levels have been reported in AS the role of MIF in bone formation has been a subject of controversy.

Objectives: Detection the level of Migration Inhibitory Factor in Ankylosing Spondylitis patients and correlate the level with disease activity and progression. Methods: This cross-sectional case-control study were concluded upon 70 randomly selected people from internal medicine department in Kobry El-kobba Military Hospital and from rheumatology and rehabilitation department in EL-Hussein university hospital. The population study were divided into two groups: Group A: (50) Patients with Ankylosing Spondylitis (AS) who met the European Spondyloarthropathy Study Group (ESSG) criteria for AS (30) and Group B: (20) Healthy controls. Serum MIF by ELISA were measured. X-rays cervical \&lumbosacral vertebrae (dorsal, lateral) views were obtained. m-SASS score (based on radiographic findings to detect progression) were assessed. MSK U/S on Tendo Achilles affected in AS patients were done.

Results: the mean age of all patients was $(38.2 \pm 8.4)$ years. Regarding gender of the patients, the majority $(65.7 \%)$ of patients were males, while $(34.3 \%)$ were females. $(88 \%)$ of AS patients had cervical erosion or sclerosis, $(46 \%)$ had dorsal erosion or sclerosis, (92\%) had lumbosacral erosion or sclerosis, with m-SASS score of $(11 \pm 6.1)$. Regarding musculoskeletal U/S, $(72 \%)$ had evidence of inflammation and erosion. Highly significant increase in ESR, CRP and MIF levels in A group; compared to control B group; with highly significant statistical difference $(p<0.01)$. the increase in smoking, ESR and MIF level; had an independent effect on increasing the probability of progression occurrence; with significant statistical difference ( $p<0.05$ respectively). MIF level at a cutoff point $(>51)$ predicted patients with progression, with fair accuracy $(74 \%)$, sensitivity= $53 \%$ and specificity $=94 \%(p=0.0056)$

Conclusion: MIF ply an important role in the pathogenesis of Ankylosing Spondylitis and has a link between chronic inflammation, new bone formation and disease progression and can be used as indicator for activity and disease progression.

Disclosure of Interests: None declared

DOI: 10.1136/annrheumdis-2021-eular.3421

\section{SLE, Sjögren's and APS - aetiology, pathogenesis and animal models}

\section{AB0077 ASSOCIATION BETWEEN VITAMIN D RECEPTOR GENE POLYMORPHISMS AND SYSTEMIC LUPUS ERYTHEMATOSUS IN MALTESE PATIENTS}

R. Magro ${ }^{1}$, J. A. Grech Meli ${ }^{2}$, J. Debattista3, N. Aquilina ${ }^{4}$, K. Gatt5, A. Borg6, C. Scerri ${ }^{2}{ }^{1}$ Mater Dei Hospital, Rheumatology, Msida, Malta; ${ }^{2}$ University of Malta, Faculty of Medicine and Surgery, Msida, Malta; ${ }^{2}$ University of Malta, Faculty of Medicine and Surgery, Msida, Malta; ${ }^{4}$ Mater Dei Hospital, Medicine, Msida, Malta; ${ }^{4}$ Mater Dei Hospital, Medicine, Msida, Malta; ${ }^{1}$ Mater Dei Hospital, Rheumatology, Msida, Malta

Background: Vitamin $D$ deficiency is highly prevalent in patients with systemic lupus erythematosus (SLE). Vitamin D acts through the vitamin D receptor (VDR) that is present in most cells, and it can regulate the transcription of over 200 genes. The expression of vitamin $D$ receptors by a variety of cells belonging to the innate and adaptive immune systems has created interest with regards to the role of vitamin $D$ in the pathogenesis of SLE. Several polymorphisms of the VDR gene have been described, namely Bsml, Apal, Taql and Fokl. A number of VDR gene polymorphism genotypes have been associated with increased risk of SLE mostly in Asians and Africans. ${ }^{1}$

Objectives: The aim of this study was to establish whether an association was present between VDR gene polymorphisms and SLE susceptibility in a cohort of SLE patients living in Malta. A further aim was to assess the relationship between these VDR gene polymorphisms and SLE disease characteristics.

Methods: 59 SLE patients living in Malta and attending Rheumatology clinic at Mater Dei Hospital were recruited for the study after providing informed consent. The patients were over the age of 18 years and fulfilled the SLICC classification criteria for SLE. The patients were interviewed and blood samples were taken. RNA extraction was performed from whole blood. QuantiGene Plex technology was used to measure the expression of 12 interferon (IFN) signature genes in the extracted RNA. 93 cord blood samples obtained from individuals living in Malta were used as a control. DNA extraction was carried out from the blood samples obtained from the patients and controls. The VDR gene was screened and the regions containing the VDR polymorphisms were amplified for each patient. The amplified regions were then digested with their respective restriction enzymes in order to view the patient's genotype via restriction fragment length polymorphism. Statistical analysis, including odds ratio (OR), was carried out to gauge the significance in the association of these polymorphisms with SLE.

Results: $94.9 \%$ of SLE patients were female and they had a mean age of 44.5 years. All the patients were of Caucasian ethnicity. $13.6 \%$ had vitamin $D$ deficiency (serum 25-hydroxyvitamin $\mathrm{D}<20 \mathrm{ng} / \mathrm{ml}$ ) and $25.4 \%$ were vitamin $\mathrm{D}$ insufficien (serum 25-hydroxyvitamin D 20-29ng/ml). The results showed that when Apal polymorphism was present as a homozygote for the variant allele there was a significant decrease in SLE prevalence $(\mathrm{OR}=0.39, \mathrm{Cl} 0.17-0.87, \mathrm{p}=0.02)$. The results were also analysed by placing the polymorphs into haplotypes. The haplotype containing all wild-type alleles for the VDR gene and the haplotype containing all wild-type alleles with the variant allele for Fokl had an increased prevalence of SLE $(\mathrm{OR}=1.95, \mathrm{Cl} 1.12-3.38, \mathrm{p}=0.01$ and $\mathrm{OR}=2.36, \mathrm{Cl} 1.13-4.91, \mathrm{p}=0.02$ respectively). The patients who were homozygous for the variant allele for $\mathrm{Bsml}$ had a significantly higher SLE disease activity index-2K (SLEDAI-2K) (mean 5.00) compared to those that were heterozygous (mean 2.66; $p=0.010$ ). No significant difference was noted in damage, IFN signature gene expression, organ manifestation and autoantibody profile between the different genotypes for the 4 VDR polymorphisms. SLE patients who were homozygous variant for the Apal or Taql polymorphisms had an increased prevalence of fibromyalgia ( $\mathrm{OR}=7.50, \mathrm{Cl} 1.47-$ 38.16, $p=0.02$ and $O R=12.00, \mathrm{Cl} 1.80-80.05, p=0.02$ respectively).

Conclusion: The study showed that in the Maltese population the presence of the VDR gene polymorphism haplotype containing all wild-type alleles and the haplotype containing all wild-type alleles with the variant allele for Fokl are associated with an increased risk of SLE. Moreover the homozygous variant genotype for Bsml was associated with a higher SLE disease activity. The homozygous 
variant genotype for Apal and Taql was associated with a higher risk of fibromyalgia in SLE patients.

REFERENCES:

[1] Zhou TB, Jiang ZP, Lin ZJ, Su N. Association of vitamin D receptor gene polymorphism with the risk of systemic lupus erythematosus. J Recept Signal Transduct Res. 2015;35(1):8-14.

Acknowledgements: The Faculty of Medicine and Surgery, University of Malta provided funding for this research.

Disclosure of Interests: None declared

DOI: 10.1136/annrheumdis-2021-eular.770

\section{AB0078 $\quad$ LIPID IMMOBILIZATION AS A METHOD TO OBTAIN ANTIGENIC NANO-OBJECTS}

O. Rusanova ${ }^{1}$, O. Emelyanova ${ }^{1,2}$, N. Emelyanov ${ }^{2} .{ }^{1}$ Federal State Budgetary Institution «Research Institute of Clinical and Experimental Rheumatology named after A.B. Zborovsky», 1, Волгоград, Russian Federation; ${ }^{2}$ Federal State Budgetary Educational Institution of Higher Education «Volgoorad State Medical University» of the Ministry of Healthcare of the Russian Federation, 2, Волгоград, Russian Federation

Objectives: Objective of the study is to research the effect of emulsion polymerization on active sites of cardiolipin antigen determinant in antiphospholipid syndrome (APS) in patients with systemic lupus erythematosus (SLE).

Methods: Having integrated antigen nanoobjects we developed immobilized magnetocontrollable antigen nanosystems and put them to an evaluation test. The nanosystems are polyacrylamide granules with a built in antigen. To obtain stable immobilized multi-use biopharmaceuticals with targeted properties (shape, particle diameter, pore size, density) we used a modified version of emulsion polymerization method using polyacrylamide carrier gel. This method permitted a greater sorptive capacity, preserving the antigen in maximum native state, and opened up the possibility of controllable modification of nanoobjects. Cardiolipin was used as the antigen in question.

Results: Following the method described above we performed sorption of anticardiolipin antibodies from blood plasma of SLE patients who showed clinical presentations of antiphospholipid syndrome. Blood serum from 10 apparently healthy individuals served as control. The level of cardiolipin antibodies was determined before and after sorption by indirect solid phase immunoenzyme method. In the eluate we estimated total protein by Lowry method. In vitro testing showed that the obtained antigen nanosystems based on immobilized cardiolipin could effectively remove cardiolipin antibodies from whole blood of SLE patients with clinical presentations of APS to achieve the values of healthy individuals (before sorption cardiolipin antibodies $0.328 \pm 0.028$; after sorption $0.059 \pm$ $0.017 ; p<0.001$; sorption capacity $8.00 \pm 0.390 \mathrm{mg} / \mathrm{ml}$ ).

Conclusion: The method of emulsion polymerization with consideration to hydrophobic and hydrophilic properties of lipid molecules permits obtaining and modifying biomolecules with certain properties, in a controlled fashion.

Disclosure of Interests: None declared

DOI: 10.1136/annrheumdis-2021-eular.1075

\section{AB0079 ENDOTHELIAL FUNCTION IN SYSTEMIC LUPUS ERYTHEMATOSUS PATIENTS: IMPACT OF CAFFEINE CONSUMPTION ON ENDOTHELIAL PROGENITOR CELLS}

V. Orefice ${ }^{1}$, F. Ceccarelli ${ }^{1}$, C. Barbati ${ }^{1}$, E. Putro ${ }^{1}$, C. Pirone ${ }^{1}$, F. R. Spinelli ${ }^{1}$, C. Alessandri', F. Conti'. 'Lupus Clinic, Sapienza University of Rome, Department of Clinical Internal, Anesthesiological and Cardiovascular Sciences, Rome, Italy

Background: As widely demonstrated, circulating endothelial progenitor cells (EPCs) could be considered biomarkers of endothelial dysfunction. Their frequency and function varied in systemic lupus erythematosus (SLE) patients, with a significant association with subclinical atherosclerosis ${ }^{1}$. Caffeine, one of the most widely consumed products in the world, seems to interact with multiple components of the immune system by acting as a non-specific phosphodiesterase inhibitor $^{2}$. In terms of cardiovascular disease (CVD), data from the literature showed a U-shaped association between habitual coffee intake and $C V D^{3}$. In this view, Spyridopoulos et al. demonstrated a significant improvement in mature endothelial cells and EPCs migration in relation to coffee consumption in coronary artery disease both in mouse models and in patients ${ }^{4}$. Finally, caffeine seems to play a positive effect on SLE disease activity status, as demonstrated by the inverse association between its intake and SLE Disease Activity Index 2000 (SLEDAl-2K) and the serum levels of inflammatory cytokines ${ }^{5}$. At the best of our knowledge, there are no data about the effect of caffeine on cardiovascular risk in SLE patients. Objectives: The aim of this study was to evaluate the possible role of caffeine intake on endothelial function in SLE patients, by evaluating its effect on circulating EPCs.
Methods: We performed a cross-sectional study enrolling SLE patients, fulfilling the revised 1997 ACR criteria. According with the protocol study, we excluded patients with history of smoking, CVD, chronic kidney failure, dyslipidaemia, and/or diabetes. At recruitment, the clinical and laboratory data were collected and disease activity was assessed using the SLEDAl-2k. Caffeine intake was evaluated using a 7-day food frequency questionnaire, previously employed in SLE cohort ${ }^{5}$. At the end of questionnaire filling, blood samples were collected. EPCs were isolated from peripheral blood mononuclear cells (PBMC) by a flow cytometry analysis and they were defined as early EPCs CD34+KDR+CD133+ cells and late EPCs CD34+KDR+CD133-, expressed as a percentage within the lymphocyte gate.

Results: We enrolled 19 patients (F:M 18:1, median age 45 years, IQR 15 median disease duration 240 months, IQR 168). In this cohort, we observed a mean \pm SD SLEDAI- $2 \mathrm{k}$ value of $1.3 \pm 3.3$ and the most frequent disease-related feature was joint involvement $(73.7 \%)$. Concerning treatment at the time of enrolment, the majority of patients were receiving treatment with hydroxychloroquine $(78.9 \%)$ and seven with glucocorticoids $(36.8 \%)$. The median intake of caffeine was $163 \mathrm{mg} /$ day (IQR 138) and we used this value as cut-off to categorize SLE patients in 2 groups: group $1(\mathrm{~N}=10$, caffeine intake $\leq 163 \mathrm{mg} /$ day) and group 2 $(\mathrm{N}=9$, caffeine intake $>163 \mathrm{mg} /$ day). Patients with less intake of caffeine showed a significantly more frequent history of lupus nephritis $(p=0.03)$, haematological manifestations $(p=0.0003)$ and anti-dsDNA positivity $(p=0.0003)$. Moving on EPCs, a positive correlation between caffeine intake and EPCs percentage was observed $(p=0.04, r=0.4)$ (Figure $1 A)$. Moreover, patients with more caffeine intake showed higher levels of early EPCs ( $p=0.02)$ (Figure 1B)

Conclusion: This is the first report analysing the impact of caffeine on EPCs frequency in SLE patients. We found a positive correlation between its intake and both early and late EPCs percentage, suggesting a caffeine influence on endothelial function in SLE patients. Nonetheless, these results support the possible impact of dietary habits on autoimmune diseases.

\section{REFERENCES:}

[1] Westerweel et al. Ann Rheum Dis 2007.

[2] Aronsen et al. Europ Joul of Pharm 2014.

[3] Ding et al. Circulation 2015

[4] Spyridopoulos et al. Art. Thromb Vasc Biol. 2008.

[5] Orefice et al. Lupus 2020.

Figure 1. A. Correlation between $K D R+C D 34+$ cells and daily caffeine intake B. $K D R+C D 34+C D 133+$ cells percentage in group $1(\mathrm{~N}=10$, caffeine intake $\leq 163 \mathrm{mg} /$ day) and group 2 $(\mathrm{N}=9$, caffeine intake $>163 \mathrm{mg} / \mathrm{day})$
A

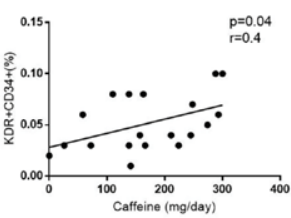

B

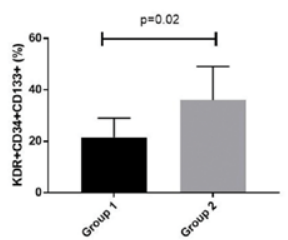

Disclosure of Interests: None declared

DOI: 10.1136/annrheumdis-2021-eular.1224

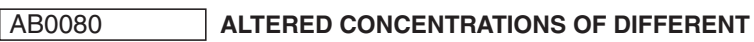 SMALL EXTRACELLULAR VESICLE POPULATIONS IN PLASMA OF PATIENTS WITH ANTIPHOSPHOLIPID SYNDROME}

U. Stok $^{1,2}$, A. Shephard ${ }^{3}$, S. Cucnik ${ }^{1,2}$, S. Sodin-Šemrl ${ }^{1,4}$, P. Zigon ${ }^{1,4}{ }^{1}{ }^{1}$ University Medical Centre Ljubljana, Department of Rheumatology, Ljubljana, Slovenia; ${ }^{2}$ University of Ljubljana, Faculty of Pharmacy, Ljubljana, Slovenia; ${ }^{3}$ Nanoview Biosciences, Research and Development, Malvern, United Kingdom; ${ }^{4}$ University of Primorska, Faculty of Mathematics, Natural Sciences and Information Technologies, Koper, Slovenia

Background: Antiphospholipid syndrome (APS) is a systemic autoimmune disorder characterized by thrombosis, obstetric complications, and the presence of antiphospholipid antibodies (aPL) that cause endothelial injury and thrombophilia [1]. Extracellular vesicles (EVs) are involved in various thrombotic disorders [2], including APS [3,4], and therefore may influence the prothrombotic status of APS patients. One of the hallmarks of activated endothelium is the expression of adhesion molecules, such as ICAM-1 (CD54) and E-selectin (CD62E), that play a key function in the interactions with leukocytes and platelets.

Objectives: To determine the level of total tetraspanin (CD81/CD63/CD9)-positive vesicles and specific EV populations (CD54- and CD62E-positive EVs) in plasma from APS patients.

Methods: Whole blood was collected from 4 APS patients and 3 healthy blood donors (HBDs) and processed to obtain platelet-depleted plasma. The size and concentration of EVs were determined using ExoView platform (NanoView 